\title{
Dibaryons with two heavy quarks
}

\author{
S.M. Gerasyuta* and E.E. Matskevich \\ Department of Theoretical Physics, St. Petersburg State University, 198904, St. Petersburg, Russia and \\ Department of Physics, LTA, 194021, St. Petersburg, Russia
}

\begin{abstract}
The relativistic six-quark equations are constructed in the framework of the dispersion relation technique. The relativistic six-quark amplitudes of dibaryons including the light $u, d$ and heavy $c$, $b$ quarks are calculated. The approximate solutions of these equations using the method based on the extraction of leading singularities of the heavy hexaquark amplitudes are obtained. The poles of these amplitudes determine the masses of charmed and bottom dibaryons with the isospins $I=0$, 1,2 and the spin-parities $J^{P}=0^{+}, 1^{+}, 2^{+}$.

PACS numbers: 11.55.Fv, 12.39.Ki, 12.39.Mk, 12.40.Yx.
\end{abstract}

\section{INTRODUCTION.}

In 1977, Jaffe [1] studied the color-magnetic interaction of the one-gluon-exchange potential in the multiquark system and found that the most attractive channel is the flavor singlet with quark content $u^{2} d^{2} s^{2}$. The same symmetry analysis of the chiral boson exchange potential leads to the similar result [2].

The $H$-particle, $N \Omega$-state and di- $\Omega$ may be strong interaction stable. Up to now, these three interesting candidates of dibaryons are still not found or confirmed by experiments. It seems that one should go beyond these candidates and should search for the possible candidates in a wider region, expecially the systems with heavy quarks, in terms of a more reliable model.

There were a number of theoretical predictions by using various models: the quark cluster model [3, 4], the quarkdelocation model [5, 6], the chiral $S U(3)$ quark model [7], the flavor $S U(3)$ skyrmion model [8]. Lomon predicted a deuteronlike dibaryon resonance using R-matrix theory [9]. By employing the chiral $S U(3)$ quark model Zhang and Yu studied $\Omega \Omega$ and $\Sigma \Omega$ states [10, 11]. Lee and Yasui discuss the stable multiquark states containing charm and bottom quark $[12]$.

In a series of papers [13-17] a method has been developed which is convenient for analyzing relativistic three-hadron systems. The physics of the three-hadron system can be described by means of a pair interaction between the particles. There are three isobar channels, each of which consists of a two-particle isobar and the third particle. The presence of the isobar representation together with the condition of unitarity in the pair energies and of analyticity leads to a system of integral equations in a single variable. Their solution makes it possible to describe the interaction of the produced particles in three-hadron systems.

In our papers [18-20] relativistic generalization of the three-body Faddeev equations was obtained in the form of dispersion relations in the pair energy of two interacting quarks. The mass spectrum of $S$-wave baryons including $u, d$, $s$ quarks was calculated by a method based on isolating the leading singularities in the amplitude. We searched for the approximate solution of integral three-quark equations by taking into account two-particle and triangle singularities, and defined all the smooth functions of the subenergy variables (as compared with the singular part of the amplitude) in the middle point of the physical region of Dalitz-plot, then the problem was reduced to the one of solving a system of simple algebraic equations.

In the present paper the relativistic six-quark equations are found in the framework of coupled-channel formalism. We use only planar diagrams; the other diagrams due to the rules of $1 / N_{c}$ expansion [21 [23] are neglected. The six-quark amplitudes of dibaryons with two heavy quarks are calculated. The poles of these amplitudes determine the masses of heavy dibaryons.

In Sec. II, the six-quark amplitudes of hexaquarks are constructed. The dynamical mixing between the subamplitudes of dibaryons are considered. The relativistic six-quark equations are constructed in the form of the dispersion relation over the two-body subenergy. The approximate solutions of these equations using the method based on the extraction of leading singularities of the amplitude are obtained. Sec. III is devoted to the calculation results for the dibaryon mass spectra (Tables I, II). In conclusion, the status of the considered model is discussed.

\footnotetext{
*Electronic address: gerasyuta@SG6488.spb.edu

${ }^{\dagger}$ Electronic address: matskev@pobox.spbu.ru
} 


\section{SIX-QUARK AMPLITUDES OF THE HEXAQUARKS WITH THE TWO HEAVY QUARKS.}

We derive the relativistic six-quark equations in the framework of the dispersion relation technique. We use only planar diagrams; the other diagrams due to the rules of $1 / N_{c}$ expansion 21 23] are neglected. The current generates a six-quark system. The correct equations for the amplitude are obtained by taking into account all possible subamplitudes. Then one should represent a six-particle amplitude as a sum of 15 subamplitudes:

$$
A=\sum_{\substack{i<j \\ i, j=1}}^{6} A_{i j} .
$$

This defines the division of the diagrams into groups according to the certain pair interaction of particles. The total amplitude can be represented graphically as a sum of diagrams. We need to consider only one group of diagrams and the amplitude corresponding to them, for example $A_{12}$. We shall consider the derivation of the relativistic generalization of the Faddeev-Yakubovsky approach. In our case, the low-lying dibaryons with the two heavy quarks are considered. We take into account the pairwise interaction of all six quarks in the hexaquark.

For instance, we consider the state $\Sigma_{c} \Sigma_{c}$ with the isospin $I=2$ and the spin-parity $J^{P}=0^{+}$(uuc uuc). The set of diagrams associated with the amplitude $A_{12}$ can further be broken down into seven groups corresponding to subamplitudes: $A_{1}^{1^{u u}}, A_{1}^{1^{c c}}, A_{1}^{0^{u c}}, A_{2}^{1^{u u}} 1^{u u}, A_{2}^{1^{u u}} 0^{u c}, A_{2}^{0^{u c}} 0^{u c}, A_{3}^{1^{u u}} 1^{u u} 1^{c c}$.

The system of graphical equations (see for example equation for the amplitude $A_{2}^{0^{u c} 0^{u c}}$ for the state $\Sigma_{c} \Sigma_{c}$ with the isospin $I=2$ and the spin-parity $J^{P}=0^{+}(u u c u u c)$ ) is determined by the subamplitudes using the self-consistent method. The coefficients are determined by the permutation of quarks.

In order to represent the subamplitudes $A_{1}^{1^{u u}}, A_{1}^{1^{c c}}, A_{1}^{0^{u c}}, A_{2}^{1^{u u}} 1^{u u}, A_{2}^{1^{u u} 0^{u c}}, A_{2}^{0^{u c} 0^{u c}}, A_{3}^{1^{u u}} 1^{u u} 1^{c c}$ in the form of a dispersion relation, it is necessary to define the amplitude of $q q, q Q$ and $Q Q$ interactions. We use the results of our relativistic quark model [24] and write down the pair quark amplitudes in the form:

$$
\begin{gathered}
a_{n}\left(s_{i k}\right)=\frac{G_{n}^{2}\left(s_{i k}\right)}{1-B_{n}\left(s_{i k}\right)}, \\
B_{n}\left(s_{i k}\right)=\int_{\left(m_{i}+m_{k}\right)^{2}}^{\frac{\left(m_{i}+m_{k}\right)^{2} \Lambda}{4}} \frac{d s_{i k}^{\prime}}{\pi} \frac{\rho_{n}\left(s_{i k}^{\prime}\right) G_{n}^{2}\left(s_{i k}^{\prime}\right)}{s_{i k}^{\prime}-s_{i k}}, \\
\rho_{n}\left(s_{i k}, J^{P}\right)=\left(\alpha\left(n, J^{P}\right) \frac{s_{i k}}{\left(m_{i}+m_{k}\right)^{2}}+\beta\left(n, J^{P}\right)+\delta\left(n, J^{P}\right) \frac{\left(m_{i}-m_{k}\right)^{2}}{s_{i k}}\right) \\
\times \frac{\sqrt{\left(s_{i k}-\left(m_{i}+m_{k}\right)^{2}\right)\left(s_{i k}-\left(m_{i}-m_{k}\right)^{2}\right)}}{s_{i k}} .
\end{gathered}
$$

The coefficients $\alpha\left(n, J^{P}\right), \beta\left(n, J^{P}\right)$ and $\delta\left(n, J^{P}\right)$ are given in Table 11 Here $n=1$ coresponds to $q q, q Q$ and $Q Q$-pairs with $J^{P}=0^{+}, n=2$ corresponds to $q q, q Q$ and $Q Q$ with $J^{P}=1^{+}$.

The coupled integral equations correspond to Fig. 1 can be described similar to 25 .

Then we can go from the integration of the cosine of the angles $d z_{i}$ to the integration over the subenergies.

Let us extract two- and three-particle singularities in the amplitudes $A_{1}^{1^{u u}}, A_{1}^{\mathbb{1}^{c c}}, A_{1}^{0^{u c}}, A_{2}^{1^{u u}} 1^{u u}, A_{2}^{1^{u u}} 0^{u c}, A_{2}^{0^{u c}} 0^{u c}$, $A_{3}^{1 u u} 1^{u u} 1^{c c}:$

$$
\begin{aligned}
& A_{1}^{1^{u u}}\left(s, s_{12345}, s_{1234}, s_{123}, s_{12}\right)=\frac{\alpha_{1}^{u u}\left(s, s_{12345}, s_{1234}, s_{123}, s_{12}\right) B_{1 u u}\left(s_{12}\right)}{\left[1-B_{1 u u}\left(s_{12}\right)\right]}, \\
& A_{1}^{1^{c c}}\left(s, s_{12345}, s_{1234}, s_{123}, s_{12}\right)=\frac{\alpha_{1}^{\alpha^{c c}}\left(s, s_{12345}, s_{1234}, s_{123}, s_{12}\right) B_{1^{c c}}\left(s_{12}\right)}{\left[1-B_{1^{c c}}\left(s_{12}\right)\right]} \\
& A_{1}^{0^{u c}}\left(s, s_{12345}, s_{1234}, s_{123}, s_{12}\right)=\frac{\alpha_{1}^{0^{u c}}\left(s, s_{12345}, s_{1234}, s_{123}, s_{12}\right) B_{0^{u c}}\left(s_{12}\right)}{\left[1-B_{0^{u c}}\left(s_{12}\right)\right]}
\end{aligned}
$$




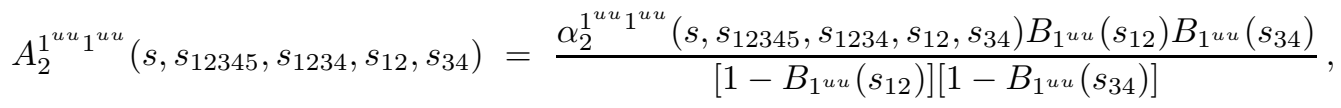

$$
\begin{aligned}
& A_{2}^{1^{u u} 0^{u c}}\left(s, s_{12345}, s_{1234}, s_{12}, s_{34}\right)=\frac{\alpha_{2}^{1^{u u} 0^{u c}}\left(s, s_{12345}, s_{1234}, s_{12}, s_{34}\right) B_{1 \text { uu }}\left(s_{12}\right) B_{0^{u c}}\left(s_{34}\right)}{\left[1-B_{1^{u u}}\left(s_{12}\right)\right]\left[1-B_{0^{u c}}\left(s_{34}\right)\right]} \\
& A_{2}^{0^{u c} 0^{u c}}\left(s, s_{12345}, s_{1234}, s_{12}, s_{34}\right)=\frac{\alpha_{2}^{0^{u c} 0^{u c}}\left(s, s_{12345}, s_{1234}, s_{12}, s_{34}\right) B_{0^{u c}}\left(s_{12}\right) B_{0^{u c}}\left(s_{34}\right)}{\left[1-B_{0^{u c}}\left(s_{12}\right)\right]\left[1-B_{0^{u c}}\left(s_{34}\right)\right]}, \\
& A_{3}^{1^{u u} 1^{u u} 1^{c c}}\left(s, s_{12345}, s_{12}, s_{34}, s_{56}\right)=\frac{\alpha_{3}^{1^{u u} 1^{u u} 1^{c c}}\left(s, s_{12345}, s_{12}, s_{34}, s_{56}\right) B_{1^{u u}}\left(s_{12}\right) B_{1^{u u}}\left(s_{34}\right) B_{1^{c c}}\left(s_{56}\right)}{\left[1-B_{1^{u u}}\left(s_{12}\right)\right]\left[1-B_{1^{u u}}\left(s_{34}\right)\right]\left[1-B_{1^{c c}}\left(s_{56}\right)\right]} .
\end{aligned}
$$

We used the classification of singularities, which was proposed in paper [26]. Using this classification, one defines the reduced amplitudes $\alpha_{1}, \alpha_{2}, \alpha_{3}$ as well as the $B$-functions in the middle point of physical region of Dalitz-plot at the point $s_{0}$.

Such choice of point $s_{0}$ allows us to replace integral equations $\left(\Sigma_{c} \Sigma_{c}, I=2, J^{P}=0^{+}\right)$by the algebraic equations (12) - (18):

$$
\begin{aligned}
& \alpha_{1}^{1^{u u}}=\lambda+4 \alpha_{1}^{1^{u u}} I_{1}\left(1^{u u} 1^{u u}\right)+4 \alpha_{1}^{0^{u c}} I_{1}\left(1^{u u} 0^{u c}\right)+2 \alpha_{2}^{1^{u u}} 1^{u u} I_{2}\left(1^{u u} 1^{u u} 1^{u u}\right)+8 \alpha_{2}^{1^{u u}} 0^{u c} I_{2}\left(1^{u u} 1^{u u} 0^{u c}\right) \\
& +2 \alpha_{2}^{0^{u c} 0^{u c}} I_{2}\left(1^{u u} 0^{u c} 0^{u c}\right) \\
& \alpha_{1}^{1^{c c}}=\lambda+8 \alpha_{1}^{0^{u c}} I_{1}\left(1^{s s} 0^{u c}\right)+12 \alpha_{2}^{0^{u c} 0^{u c}} I_{2}\left(1^{c c} 0^{u c} 0^{u c}\right) \\
& \alpha_{1}^{0^{u c}}=\lambda+3 \alpha_{1}^{1^{u u}} I_{1}\left(0^{u c} 1^{u u}\right)+\alpha_{1}^{1^{c c}} I_{1}\left(0^{u c} 1^{c c}\right)+4 \alpha_{1}^{0^{u c}} I_{1}\left(0^{u c} 0^{u c}\right)+6 \alpha_{2}^{1^{u u}} 0^{u c} I_{2}\left(0^{u c} 1^{u u} 0^{u c}\right) \\
& +3 \alpha_{2}^{0^{u c} 0^{u c}} I_{2}\left(0^{u c} 0^{u c} 0^{u c}\right) \\
& \alpha_{2}^{1^{u u} 1^{u u}}=\lambda+4 \alpha_{1}^{1^{u u}} I_{3}\left(1^{u u} 1^{u u} 1^{u u}\right)+8 \alpha_{1}^{0^{u c}} I_{4}\left(1^{u u} 1^{u u} 0^{u c}\right)+16 \alpha_{2}^{1^{u u}} 0^{u c} I_{7}\left(1^{u u} 1^{u u} 1^{u u} 0^{u c}\right) \\
& +\alpha_{2}^{0^{u c} 0^{u c}}\left(4 I_{5}\left(1^{u u} 1^{u u} 0^{u s} 0^{u c}\right)+8 I_{6}\left(1^{u u} 1^{u u} 0^{u c} 0^{u c}\right)\right) \\
& \alpha_{2}^{1^{u u} 0^{u c}}=\lambda+\alpha_{1}^{1^{u u}}\left(2 I_{3}\left(1^{u u} 0^{u c} 1^{u u}\right)+I_{4}\left(0^{u c} 1^{u u} 1^{u u}\right)\right)+\alpha_{1}^{0^{u c}}\left(2 I_{3}\left(1^{u u} 0^{u c} 0^{u c}\right)+2 I_{4}\left(1^{u u} 0^{u c} 0^{u c}\right)\right) \\
& +\alpha_{2}^{1 u u} 0^{u c}\left(2 I_{5}\left(1^{u u} 0^{u c} 1^{u u} 0^{u c}\right)+2 I_{6}\left(1^{u u} 0^{u s} 0^{u c} 1^{u u}\right)+2 I_{7}\left(1^{u u} 0^{u c} 1^{u u} 0^{u c}\right)+2 I_{7}\left(1^{u u} 0^{u s} 0^{u c} 1^{u u}\right)\right. \\
& \left.+2 I_{7}\left(0^{u c} 1^{u u} 1^{u u} 0^{u c}\right)\right)+\alpha_{2}^{0^{u c}} 0^{u c}\left(I_{5}\left(0^{u c} 1^{u u} 0^{u c} 0^{u c}\right)+2 I_{6}\left(1^{u u} 0^{u c} 0^{u c} 0^{u c}\right)+2 I_{7}\left(0^{u c} 1^{u u} 0^{u c} 0^{u c}\right)\right) \\
& \alpha_{2}^{0^{u c} 0^{u c}}=\lambda+\alpha_{1}^{1^{u u}}\left(I_{3}\left(0^{u c} 0^{u c} 1^{u u}\right)+4 I_{4}\left(0^{u c} 0^{u c} 1^{u u}\right)\right)+\alpha_{1}^{1^{c c}} I_{3}\left(0^{u c} 0^{u c} 1^{c c}\right)+\alpha_{1}^{0^{u c}}\left(2 I_{3}\left(0^{u c} 0^{u c} 0^{u c}\right)\right. \\
& \left.+4 I_{4}\left(0^{u c} 0^{u c} 0^{u c}\right)\right)+2 \alpha_{2}^{1^{u u}} 1^{u u} I_{6}\left(0^{u c} 0^{u c} 1^{u u} 1^{u u}\right)+\alpha_{2}^{1^{u u}} 0^{u c}\left(4 I_{5}\left(0^{u c} 0^{u c} 1^{u u} 0^{u c}\right)\right. \\
& \left.+4 I_{6}\left(0^{u c} 0^{u c} 1^{u u} 0^{u c}\right)+4 I_{7}\left(0^{u c} 0^{u c} 1^{u u} 0^{u c}\right)+4 I_{7}\left(0^{u c} 0^{u c} 0^{u c} 1^{u u}\right)\right)+\alpha_{2}^{0^{u c}} 0^{u c}\left(2 I_{6}\left(0^{u c} 0^{u c} 0^{u c} 0^{u c}\right)\right. \\
& \left.+4 I_{7}\left(0^{u c} 0^{u c} 0^{u c} 0^{u c}\right)\right)+2 \alpha_{3}^{1^{u u}} 1^{u u} 1^{c c} I_{8}\left(0^{u c} 0^{u c} 1^{u u} 1^{c c} 1^{u u}\right) \\
& \alpha_{3}^{1^{u u} 1^{u u} 1^{c c}}=\lambda+4 \alpha_{1}^{1^{u u}} I_{9}\left(1^{u u} 1^{u u} 1^{c c} 1^{u u}\right)+8 \alpha_{1}^{0^{u c}} I_{9}\left(1^{u u} 1^{c c} 1^{u u} 0^{u c}\right)+16 \alpha_{2}^{1^{u u} 0^{u c}} I_{10}\left(1^{u u} 1^{u u} 1^{c c} 1^{u u} 0^{u c}\right)
\end{aligned}
$$




$$
+8 \alpha_{2}^{0^{u c} 0^{u c}} I_{10}\left(1^{u u} 1^{c c} 1^{u u} 0^{u c} 0^{u c}\right)
$$

where $\lambda_{i}$ are the current constants. We used the functions $I_{1}, I_{2}, I_{3}, I_{4}, I_{5}, I_{6}, I_{7}, I_{8}, I_{9}, I_{10}$ similar to the paper [27]. The solutions of the system of equations are considered as:

$$
\alpha_{i}(s)=\frac{F_{i}\left(s, \lambda_{i}\right)}{D(s)},
$$

where zeros of $D(s)$ determinants define the masses of bound states of dibaryons.

\section{CALCULATION RESULTS.}

The model in question take into account the hexaquarks with the two heavy quarks $q q q q Q Q, q=u, d, Q=c, b$ : uuuucc, uuudcc, uuddcc, uuuubb, uuudbb, uuddbb.

The quark masses of the model are $m_{q}=495 \mathrm{MeV}, m_{c}=1655 \mathrm{MeV}$ and $m_{b}=4840 \mathrm{MeV}$.

The experimental data are absent, therefore we use the dimensionless parameters, which are similar to the previous paper [25]. It allows us to calculate the mass spectra of $q q q q Q Q$ states. We use the gluon coupling constants $g_{0}=0.653$ (diquark $J^{P}=0^{+}$) and $g_{1}=0.292$ (diquark $J^{P}=1^{+}$), cutoff parameter $\Lambda=11$. We consider the $\Lambda_{q c, c c}=8.52$, which are determined by $M=5250 \mathrm{MeV}\left(I J=22 \Sigma_{c} \Sigma_{c}^{*}, \Sigma_{c}^{*} \Sigma_{c}^{*}\right)$, the threshold is $5290 \mathrm{MeV}$. In the case of $b$-quarks the $\Lambda_{q b, b b}=7.35$ is determined by $M=11620 \mathrm{MeV}\left(I J=22 \Sigma_{b} \Sigma_{b}^{*}, \Sigma_{b}^{*} \Sigma_{b}^{*}\right)$, the threshold is $11660 \mathrm{MeV}$.

We have calculated the heavy dibaryon masses with isospin $I=0,1,2$ and spin-parity $J^{P}=0^{+}, 1^{+}, 2^{+}$, which are given in the Tables [ and [I] The relativistic six-body approach possesses the dynamical mixing and allows us to calculate the contributions of the subamplitudes to the hexaquark amplitude (Tables [V] - VI). The calculated dibaryon subamplitudes $A_{2}$ present the main contributions to the hexaquark amplitude (about 70 percents). We use only two new parameters for the calculation of 23 qqqqcc states and 19 qqqqbb states.

The lowest mass for the qqqqcc states is $M=4364 \mathrm{MeV}$, for the $q q q q b b$ states is $M=8670 \mathrm{MeV}$.

In quark models, which describe rather well the masses and static properties of hadrons, the masses of the quarks usually have the similar values for the spectra of light and heavy hadrons. However, this is achieved at the expence of some difference in the characteristic of the confinement potential. It should be borne in mind that for a fixed hadron mass the masses of the constituent quarks which enter into the composition of the hadron will become smaller when the slope of the confinement potential increases or its radius decreases. Therefore, conversely, we can change the masses of the constituent quarks when going from the spectrum of light to the heavy hadrons, while keeping the characteristic of the confinement potential unchanged. We can effectively take into account the contribution of the confinement potential in obtaining the spectrum of heavy hadrons. We neglect with the mass distinction of $u$ and $d$ quarks. The estimation of the theoretical error on the heavy dibaryons masses is $1 \mathrm{MeV}$. This result was obtained by the choice of model parameters.

\section{CONCLUSIONS.}

In a strongly bound systems, which include the light quarks, where $p / m \sim 1$, the approximation of nonrelativistic kinematics and dynamics is not justified. In our paper, the relativistic description of six-particles amplitudes of heavy dibaryons with the two heavy quarks is considered. We take into account the $u, d, c, b$ quarks. Our model is confined to the quark-antiquark pair production on account of the phase space restriction. Here $m_{q}$ and $m_{Q}$ the "mass" of the constituent quark. Therefore the production of new quark-antiquark pair is absent for the low-lying hadrons.

Hadronic molecules are loosely states of hadrons, whose inter-hadron distances are larger than the quark confinement size.

The heavy analogue of $H$ dibaryon $\left(\Lambda_{c} \Lambda_{c}\right)$ does not exist though its potential is attractive [28]. Oka et al. believe that the future studies may specify the binding energy of such a molecule state. The binding energy is sensitive to the cutoff parameter [29]. Our calculation allows us to obtain the $\Lambda_{c} \Lambda_{c}$ molecule bound state (Table I). There exist a loosely bound state with a small binding energy $E_{B}=54 \mathrm{MeV}$. In the case of $\Sigma_{c} \Sigma_{c}$ dibaryon we obtain the bound state, but the binding energy is equal to $E_{B}=540 \mathrm{MeV}$.

The similar results are obtained for the other heavy dibaryons with the two heavy quarks (Tables I- II). For the $\Lambda_{b} \Lambda_{b}$ system does not exist a loosely bound state with a small binding energy. The heavy dibaryons and heavy baryon-antibaryons may be produced at LHC. 


\section{Acknowledgments}

Gerasyuta S.M. would like to thank T. Barnes for useful discussions. The work was carried with the support of the Russian Ministry of Education (grant 2.1.1.68.26).

[1] R.L. Jaffe, Phys. Rev. Lett. 38, 195 (1977).

[2] F. Wang, J.L. Ping, H.R. Pang, and T. Goldman, Mod. Phys. Lett. A18, 356 (2003).

[3] T. Kamae and T. Fujita, Phys. Rev. Lett. 38, 471 (1977).

[4] K. Yazaki, Prog. Theor. Phys. Suppl. 91, 146 (1987).

[5] F. Wang, G.H. Wu, L.J. Teng, and T. Goldman, Phys. Rev. Lett. 69, 2901 (1992).

[6] T. Goldman, K. Maltman, G.J. Stephenson Jr, J.-L. Ping, and F. Wang, Mod. Phys. Lett. A13, 59 (1998).

[7] Z.Y. Zhang et al., Nucl. Phys. A670, 178 (2000).

[8] V.B. Kopeliovich, Nucl. Phys. A639, 75 (1998).

[9] P. LaFrance and E.L. Lomon, Phys. Rev. D34, 1341 (1986).

[10] Y.W. Yu, Z.Y. Zhang, and X.Q. Yuan, Commun. Theor. Phys. 31, 1 (1999).

[11] Y.W. Yu, Z.Y. Zhang, and X.Q. Yuan, High Energy Phys. and Nucl. Phys. 23, 859 (1999).

[12] S.H. Lee and S. Yasui, arXiv:0901.2977 [hep-ph].

[13] I.J.R. Aitchison, J. Phys. G3, 121 (1977).

[14] J.J. Brehm, Ann. Phys. (N.Y.) 108, 454 (1977).

[15] I.J.R. Aitchison and J.J. Brehm, Phys. Rev. D17, 3072 (1978).

[16] I.J.R. Aitchison and J.J. Brehm, Phys. Rev. D20, 1119 (1979).

[17] J.J. Brehm, Phys. Rev. D21, 718 (1980).

[18] S.M. Gerasyuta, Yad. Fiz. 55, 3030 (1992) [Sov. J. Nucl. Phys. 55, 1693 (1992)].

[19] S.M. Gerasyuta, Nuovo Cimento Soc. Ital. Fis. A106, 37 (1993).

[20] S.M. Gerasyuta, Z. Phys. C60, 683 (1993).

[21] G.'t Hooft, Nucl. Phys. B72, 461 (1974).

[22] G. Veneziano, Nucl. Phys. B117, 519 (1976).

[23] E. Witten, Nucl. Phys. B160, 57 (1979).

[24] V.V. Anisovich, S.M. Gerasyuta and A.V. Sarantsev, Int. J. Mod. Phys. A6, 625 (1991).

[25] S.M. Gerasyuta and E.E. Matskevich, Phys. Rev. D82, 056002 (2010).

[26] V.V. Anisovich and A.A. Anselm, Usp. Fiz. Nauk 88, 287 (1966).

[27] S.M. Gerasyuta and E.E. Matskevich, arXiv:1105.0817 v2 [hep-ph].

[28] N. Lee, Z.G. Luo, X.L. Chen, and S.L. Zhu, arXiv:1104.4257 [hep-ph].

[29] W. Meguro, Y.R. Liu, and M. Oka, arXiv:1105.3693 [hep-ph]. 
TABLE I: S-wave charmed dibaryon masses. Parameters of model: cutoff $\Lambda=11.0$ and $\Lambda_{q c, c c}=8.52$, gluon coupling constants $g_{0}=0.653$ and $g_{1}=0.292$. Quark masses $m_{q}=495 \mathrm{MeV}$ and $m_{c}=1655 \mathrm{MeV}$.

\begin{tabular}{|c|c|c|c|}
\hline$I$ & $J$ & Dibaryons (quark content) & Mass $(\mathrm{MeV})$ \\
\hline \multirow[t]{6}{*}{2} & \multirow[t]{2}{*}{0} & $\Sigma_{c} \Sigma_{c}, \Sigma_{c}^{*} \Sigma_{c}^{*}($ uис uuc $)$ & 4933 \\
\hline & & $\Delta \Xi_{c c}^{*}(u u u$ ucc $)$ & 5231 \\
\hline & \multirow[t]{2}{*}{1} & $\Sigma_{c} \Sigma_{c}, \Sigma_{c} \Sigma_{c}^{*}, \Sigma_{c}^{*} \Sigma_{c}^{*}(u u c u u c)$ & 4933 \\
\hline & & $\Delta \Xi_{c c}, \Delta \Xi_{c c}^{*}(u u u u c c)$ & 5231 \\
\hline & \multirow[t]{2}{*}{2} & $\Sigma_{c} \Sigma_{c}^{*}, \Sigma_{c}^{*} \Sigma_{c}^{*}($ uuc uuc $)$ & 5250 \\
\hline & & $\Delta \Xi_{c c}, \Delta \Xi_{c c}^{*}($ uиu ucc $)$ & 5231 \\
\hline \multirow{3}{*}{\multicolumn{2}{|c|}{$1 \mid 0$}} & $\Sigma_{c} \Sigma_{c}, \Sigma_{c}^{*} \Sigma_{c}^{*}, \Sigma_{c} \Lambda_{c}(u u c u d c)$ & 4420 \\
\hline & & $\Delta \Xi_{c c}^{*}(u u u d c c+u u d u c c)$ & 4956 \\
\hline & & $N \Xi_{c c}(u u d u c c)$ & 4956 \\
\hline \multirow{3}{*}{\multicolumn{2}{|c|}{1}} & $\Sigma_{c} \Sigma_{c}, \Sigma_{c} \Sigma_{c}^{*}, \Sigma_{c}^{*} \Sigma_{c}^{*}, \Sigma_{c} \Lambda_{c}, \Sigma_{c}^{*} \Lambda_{c}(u u c u d c)$ & 4420 \\
\hline & & $\Delta \Xi_{c c}, \Delta \Xi_{c c}^{*}(u u u d c c+u u d u c c)$ & 4956 \\
\hline & & $N \Xi_{c c}, N \Xi_{c c}^{*}(u u d u c c)$ & 4956 \\
\hline \multirow{3}{*}{\multicolumn{2}{|c|}{2}} & $\Sigma_{c} \Sigma_{c}^{*}, \Sigma_{c}^{*} \Sigma_{c}^{*}, \Sigma_{c}^{*} \Lambda_{c}(u u c u d c)$ & 4911 \\
\hline & & $\Delta \Xi_{c c}, \Delta \Xi_{c c}^{*}(u u u d c c+u u d u c c)$ & 4999 \\
\hline & & $N \Xi_{c c}^{*}(u u d u c c)$ & 5136 \\
\hline \multirow{3}{*}{\multicolumn{2}{|c|}{$0 \longdiv { 0 }$}} & $\Sigma_{c} \Sigma_{c}, \Sigma_{c}^{*} \Sigma_{c}^{*},(u u c d d c+u d c u d c)$ & 4364 \\
\hline & & $\Sigma_{c} \Lambda_{c}, \Lambda_{c} \Lambda_{c}(u d c u d c)$ & 4516 \\
\hline & & $N \Xi_{c c}, \Delta \Xi_{c c}^{*}(u u d d c c+u d d u c c)$ & 4740 \\
\hline \multirow{3}{*}{\multicolumn{2}{|c|}{1}} & $\Sigma_{c} \Sigma_{c}, \Sigma_{c} \Sigma_{c}^{*}, \Sigma_{c}^{*} \Sigma_{c}^{*},(u u c d d c+u d c u d c)$ & 4364 \\
\hline & & $\Sigma_{c} \Lambda_{c}, \Sigma_{c}^{*} \Lambda_{c} \Lambda_{c} \Lambda_{c}(u d c u d c)$ & 4516 \\
\hline & & $N \Xi_{c c}, N \Xi_{c c}^{*}, \Delta \Xi_{c c}, \Delta \Xi_{c c}^{*}(u u d d c c+u d d u c c)$ & 4740 \\
\hline \multirow{2}{*}{\multicolumn{2}{|c|}{2}} & $\Sigma_{c} \Sigma_{c}^{*}, \Sigma_{c}^{*} \Sigma_{c}^{*},(u u c d d c+u d c u d c)$ & 5086 \\
\hline & & $N \Xi_{c c}^{*}, \Delta \Xi_{c c}, \Delta \Xi_{c c}^{*}(u u d d c c+u d d u c c)$ & 5029 \\
\hline
\end{tabular}


TABLE II: S-wave bottom dibaryon masses. Parameters of model: cutoff $\Lambda=11.0$ and $\Lambda_{q b, b b}=7.35$, gluon coupling constants $g_{0}=0.653$ and $g_{1}=0.292$. Quark masses $m_{q}=495 \mathrm{MeV}$ and $m_{b}=4840 \mathrm{MeV}$.

\begin{tabular}{|c|c|c|c|}
\hline$I$ & $J$ & Dibaryons (quark content) & Mass $(\mathrm{MeV})$ \\
\hline \multirow[t]{6}{*}{2} & \multirow[t]{2}{*}{0} & $\Sigma_{b} \Sigma_{b}, \Sigma_{b}^{*} \Sigma_{b}^{*}(u u b u u b)$ & 10290 \\
\hline & & $\Delta \Xi_{b b}^{*}(u u u u b b)$ & - \\
\hline & \multirow[t]{2}{*}{1} & $\Sigma_{b} \Sigma_{b}, \Sigma_{b} \Sigma_{b}^{*}, \Sigma_{b}^{*} \Sigma_{b}^{*}(u u b u u b)$ & 10290 \\
\hline & & $\Delta \Xi_{b b}, \Delta \Xi_{b b}^{*}(u u u u b b)$ & - \\
\hline & \multirow[t]{2}{*}{2} & $\Sigma_{b} \Sigma_{b}^{*}, \Sigma_{b}^{*} \Sigma_{b}^{*}(u u b u u b)$ & 11620 \\
\hline & & $\Delta \Xi_{b b}, \Delta \Xi_{b b}^{*}(u u u u b b)$ & - \\
\hline \multirow{3}{*}{\multicolumn{2}{|c|}{$1 \mid 0$}} & $\Sigma_{b} \Sigma_{b}, \Sigma_{b}^{*} \Sigma_{b}^{*}, \Sigma_{b} \Lambda_{b}(u u b u d b)$ & 8670 \\
\hline & & $\Delta \Xi_{b b}^{*}(u u u d b b+u u d u b b)$ & 11395 \\
\hline & & $N \Xi_{b b}(u u d u b b)$ & 11395 \\
\hline \multirow{3}{*}{\multicolumn{2}{|c|}{1}} & $\Sigma_{b} \Sigma_{b}, \Sigma_{b} \Sigma_{b}^{*}, \Sigma_{b}^{*} \Sigma_{b}^{*}, \Sigma_{b} \Lambda_{b}, \Sigma_{b}^{*} \Lambda_{b}(u u b u d b)$ & 8670 \\
\hline & & $\Delta \Xi_{b}, \Delta \Xi_{b b}^{*}(u u u d b b+u u d u b b)$ & 11395 \\
\hline & & $N \Xi_{b b}, N \Xi_{b}^{*}(u u d u b b)$ & 11395 \\
\hline & \multirow[t]{3}{*}{2} & $\Sigma_{b} \Sigma_{b}^{*}, \Sigma_{b}^{*} \Sigma_{b}^{*}, \Sigma_{b}^{*} \Lambda_{b}(u u b u d b)$ & 10715 \\
\hline & & $\Delta \Xi_{b b}, \Delta \Xi_{b b}^{*}(u u u d b b+u u d u b b)$ & 11372 \\
\hline & & $N \Xi_{b b}^{*}(u u d u b b)$ & - \\
\hline \multirow{3}{*}{\multicolumn{2}{|c|}{$0 \mid 0$}} & $\Sigma_{b} \Sigma_{b}, \Sigma_{b}^{*} \Sigma_{b}^{*},(u u b d d b+u d b u d b)$ & 8482 \\
\hline & & $\Sigma_{b} \Lambda_{b}, \Lambda_{b} \Lambda_{b}(u d b u d b)$ & 9175 \\
\hline & & $N \Xi_{b b}, \Delta \Xi_{b b}^{*}(u u d d b b+u d d u b b)$ & 10828 \\
\hline \multirow{3}{*}{\multicolumn{2}{|c|}{1}} & $\Sigma_{b} \Sigma_{b}, \Sigma_{b} \Sigma_{b}^{*}, \Sigma_{b}^{*} \Sigma_{b}^{*},(u u b d d b+u d b u d b)$ & 8482 \\
\hline & & $\Sigma_{b} \Lambda_{b}, \Sigma_{b}^{*} \Lambda_{b} \Lambda_{b} \Lambda_{b}(u d b u d b)$ & 9175 \\
\hline & & $N \Xi_{b b}, N \Xi_{b b}^{*}, \Delta \Xi_{b b}, \Delta \Xi_{b b}^{*}(u u d d b b+u d d u b b)$ & 10828 \\
\hline \multirow{2}{*}{\multicolumn{2}{|c|}{2}} & $\Sigma_{b} \Sigma_{b}^{*}, \Sigma_{b}^{*} \Sigma_{b}^{*},(u u b d d b+u d b u d b)$ & 11518 \\
\hline & & $N \Xi_{b b}^{*}, \Delta \Xi_{b b}, \Delta \Xi_{b b}^{*}(u u d d b b+u d d u b b)$ & 11583 \\
\hline
\end{tabular}

TABLE III: Vertex functions and Chew-Mandelstam coefficients.

\begin{tabular}{|c|c|c|c|c|}
\hline$i$ & $G_{i}^{2}\left(s_{k l}\right)$ & $\alpha_{i}$ & $\beta_{i}$ & $\delta_{i}$ \\
\hline $0^{+}$ & $\frac{4 g}{3}-\frac{8 g m_{k l}^{2}}{\left(3 s_{k l}\right)}$ & $\frac{1}{2}$ & $-\frac{1}{2} \frac{\left(m_{k}-m_{l}\right)^{2}}{\left(m_{k}+m_{l}\right)^{2}}$ & 0 \\
$1^{+}$ & $\frac{2 g}{3}$ & $\frac{1}{3}$ & $\frac{4 m_{k} m_{l}}{3\left(m_{k}+m_{l}\right)^{2}}-\frac{1}{6}$ & $-\frac{1}{6} \frac{\left(m_{k}-m_{l}\right)^{2}}{\left(m_{k}+m_{l}\right)^{2}}$ \\
\hline
\end{tabular}


TABLE IV: $I J=00 \Sigma_{Q} \Sigma_{Q}, \Sigma_{Q}^{*} \Sigma_{Q}^{*}$. Parameters of model: cutoff $\Lambda=11.0, \Lambda_{q c, c c}=8.52, \Lambda_{q b, b b}=7.35$, gluon coupling constants $g_{0}=0.653$ and $g_{1}=0.292$. Quark masses $m_{q}=495 \mathrm{MeV}, m_{c}=1655 \mathrm{MeV}$ and $m_{b}=4840 \mathrm{MeV}$.

\begin{tabular}{|c|c|c|}
\hline Subamplitudes & \multicolumn{2}{|c|}{ Contributions, percent } \\
\cline { 2 - 3 } & $Q=c$ & $Q=b$ \\
\hline$A_{1}^{1^{u u}}$ & 2.6 & 4.6 \\
$A_{1}^{1^{d d}}$ & 2.6 & 4.6 \\
$A_{1}^{1^{Q Q}}$ & 3.7 & 6.4 \\
$A_{1}^{0^{u d}}$ & 6.4 & 10.2 \\
$A_{1}^{0^{u Q}}$ & 3.1 & 2.5 \\
$A_{1}^{0^{d Q}}$ & 3.1 & 2.5 \\
$A_{3}^{1^{u u}} 1^{d d} 1^{Q Q}$ & 2.6 & 1.8 \\
$A_{3}^{0^{u d} 0^{u Q} 0^{d Q}}$ & 2.0 & 0.2 \\
$A_{2}^{1^{u u}} 1^{d d}$ & 6.1 & 10.2 \\
$A_{2}^{1^{u u}} 0^{d Q}$ & 2.8 & 2.2 \\
$A_{2}^{1^{d d}} 0^{u Q}$ & 2.8 & 2.2 \\
$A_{2}^{0^{u d}} 0^{u d}$ & 25.4 & 35.5 \\
$A_{2}^{0^{u d}} 0^{u Q}$ & 7.4 & 5.0 \\
$A_{2}^{0^{u d}} 0^{d Q}$ & 7.4 & 5.0 \\
$A_{2}^{0^{u Q}} 0^{u Q}$ & 7.8 & 2.6 \\
$A_{2}^{0^{d Q}} 0^{d Q}$ & 7.8 & 2.6 \\
$A_{2}^{0^{u Q}} 0^{d Q}$ & 6.6 & 2.2 \\
\hline$\sum A_{1}$ & 21.4 & 30.6 \\
$\sum \sum^{d} A_{2}$ & 74.0 & 67.5 \\
$\sum A_{3}$ & 4.6 & 2.0 \\
\hline
\end{tabular}


TABLE V: $I J=00 \Sigma_{Q} \Lambda_{Q}, \Lambda_{Q} \Lambda_{Q}$. Parameters of model: cutoff $\Lambda=11.0, \Lambda_{q c, c c}=8.52, \Lambda_{q b, b b}=7.35$, gluon coupling constants $g_{0}=0.653$ and $g_{1}=0.292$. Quark masses $m_{q}=495 \mathrm{MeV}, m_{c}=1655 \mathrm{MeV}$ and $m_{b}=4840 \mathrm{MeV}$.

\begin{tabular}{|c|c|c|}
\hline Subamplitudes & \multicolumn{2}{|c|}{ Contributions, percent } \\
\cline { 2 - 3 } & $Q=c$ & $Q=b$ \\
\hline$A_{1}^{1^{u u}}$ & 3.0 & 5.6 \\
$A_{1}^{1^{d d}}$ & 3.0 & 5.6 \\
$A_{1}^{1^{Q Q}}$ & 4.3 & 8.3 \\
$A_{1}^{0^{u d}}$ & 7.0 & 11.4 \\
$A_{1}^{0^{u Q}}$ & 3.6 & 3.2 \\
$A_{1}^{0^{d Q}}$ & 3.6 & 3.2 \\
$A_{3}^{0^{u d} 0^{u Q} 0^{d Q}}$ & 3.1 & 0.3 \\
$A_{2}^{0^{u d}} 0^{u d}$ & 28.8 & 39.8 \\
$A_{2}^{0^{u d}} 0^{u Q}$ & 9.2 & 6.9 \\
$A_{2}^{0^{u d}} 0^{d Q}$ & 9.2 & 6.9 \\
$A_{2}^{0^{u Q}} 0^{u Q}$ & 10.1 & 3.6 \\
$A_{2}^{0^{d Q}} 0^{d Q}$ & 10.1 & 3.6 \\
$A_{2}^{0^{u Q}} 0^{d Q}$ & 5.1 & 1.8 \\
\hline$\sum A_{1}$ & 24.5 & 37.2 \\
$\sum A_{2}$ & 72.4 & 62.5 \\
$\sum A_{3}$ & 3.1 & 0.3 \\
\hline
\end{tabular}

TABLE VI: $I J=00 N \Xi_{Q Q}, \Delta \Xi_{Q Q}^{*}$. Parameters of model: cutoff $\Lambda=11.0, \Lambda_{q c, c c}=8.52, \Lambda_{q b, b b}=7.35$, gluon coupling constants $g_{0}=0.653$ and $g_{1}=0.292$. Quark masses $m_{q}=495 \mathrm{MeV}, m_{c}=1655 \mathrm{MeV}$ and $m_{b}=4840 \mathrm{MeV}$.

\begin{tabular}{|c|c|c|}
\hline Subamplitudes & \multicolumn{2}{|c|}{ Contributions, percent } \\
\cline { 2 - 3 } & $Q=c$ & $Q=b$ \\
\hline$A_{1}^{1^{u u}}$ & 2.1 & 1.8 \\
$A_{1}^{1^{d d}}$ & 2.1 & 1.8 \\
$A_{1}^{1^{Q Q}}$ & 3.6 & 5.7 \\
$A_{1}^{0^{u d}}$ & 6.6 & 6.2 \\
$A_{1}^{0^{u Q}}$ & 3.7 & 2.1 \\
$A_{1}^{0^{d Q}}$ & 3.7 & 2.1 \\
$A_{3}^{1^{u u} 1^{d d} 1^{Q Q}}$ & 4.9 & 4.8 \\
$A_{3}^{0^{u d} 0^{u Q} 0^{d Q}}$ & 4.9 & 0.6 \\
$A_{2}^{1^{u u}} 1^{Q Q}$ & 10.6 & 15.7 \\
$A_{2}^{1^{d d}} 1^{Q Q}$ & 10.6 & 15.7 \\
$A_{2}^{1^{u u}} 0^{d Q}$ & 3.0 & 10.0 \\
$A_{2}^{1^{d d} 0^{u Q}}$ & 3.0 & 10.0 \\
$A_{2}^{1^{Q Q}} 0^{u d}$ & 22.3 & 34.6 \\
$A_{2}^{0^{u d}} 0^{u Q}$ & 9.5 & 3.6 \\
$A_{2}^{0^{u d}} 0^{d Q}$ & 9.5 & 3.6 \\
\hline$\sum A_{1}$ & 21.7 & 19.6 \\
$\sum A_{2}$ & 68.6 & 75.1 \\
$\sum A_{3}$ & 9.8 & 5.4 \\
\hline
\end{tabular}



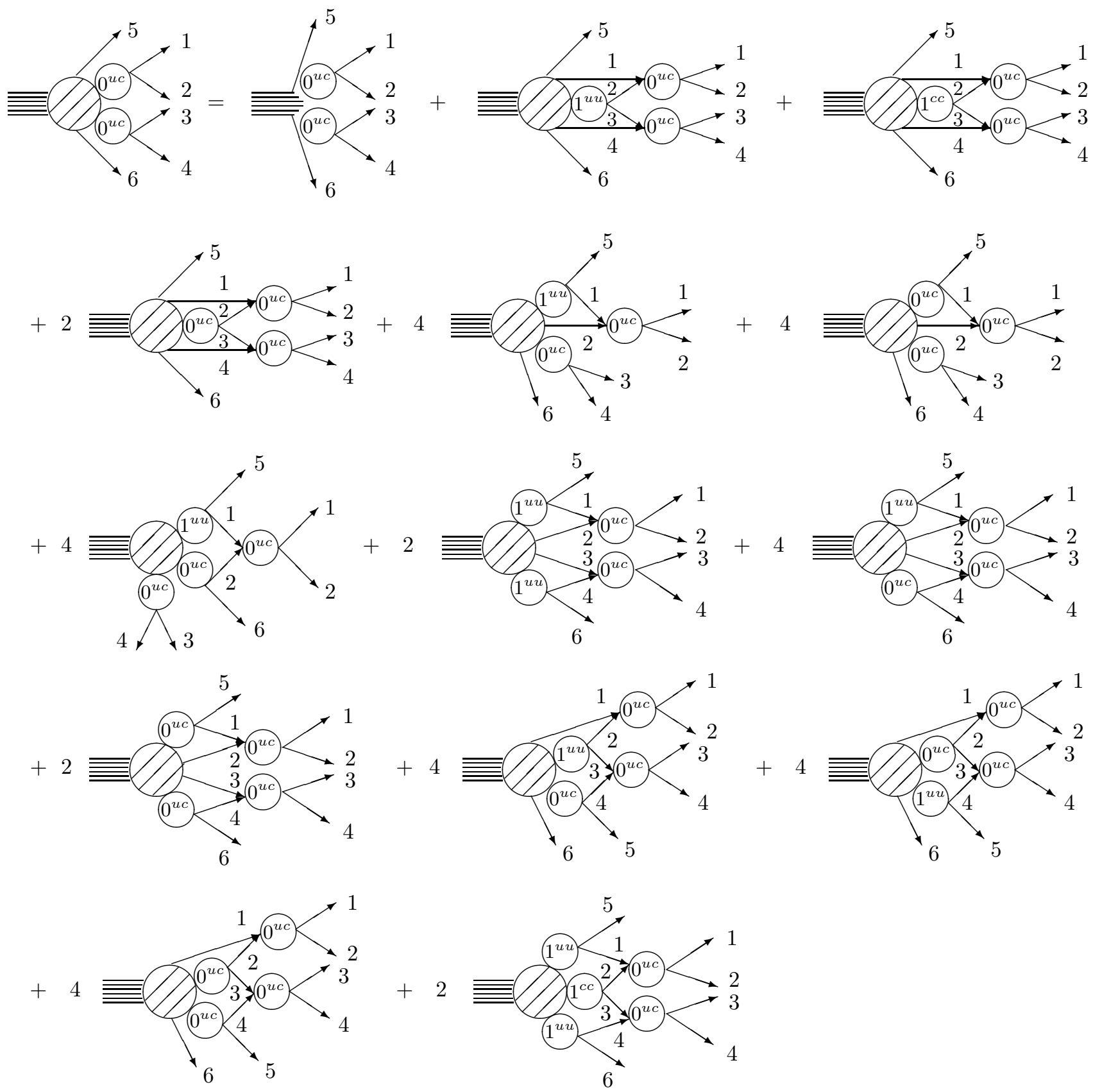

Fig. 1. Graphical representation of the amplitude $A_{2}^{0^{u c} 0^{u c}}$ for the case of $\Sigma_{c} \Sigma_{c}, \Sigma_{c}^{*} \Sigma_{c}^{*}, I=2, J^{P}=0^{+}$. 